\title{
Optimal control of myocardial ischaemia: the benefit of a fixed combination of atenolol and nifedipine in patients with chronic stable angina
}

\author{
Cardiovascular \\ Research Unit, \\ Royal Postgraduate \\ Medical School, \\ Hammersmith \\ Hospital, London \\ H El-Tamimi \\ G J Davies \\ Correspondence to: \\ Dr H El-Tamimi, \\ Division of Cardiology, \\ University of Florida College \\ of Medicine, 1600 Arch \\ Goad, PO Box 100277, \\ 0277, USA. \\ Accepted for publication \\ 20 September 1991
}

Hassan El-Tamimi, Graham J Davies induced ischaemia, the combination was more effective in reducing total ischaemic burden.

\section{(Br Heart J 1992;68:291-5)}

In most patients with chronic stable angina, both an increase in myocardial oxygen consumption and a decrease in myocardial blood flow contribute to the development of ischaemia, whether symptoms occur at rest or during exercise. ${ }^{12} \beta$ Blockers relieve ischaemia primarily by decreasing myocardial oxygen demand, and calcium channel blockers do so by improving myocardial oxygen supply. By acting on these different determinants of myocardial ischaemia, the combination of both drugs could provide better control of myocardial ischaemia.

We have investigated the anti-ischaemic effects of a calcium antagonist (nifedipine) and a $\beta$ blocker (atenolol) singly and in a fixed combination on symptoms, and exercise induced and ambulatory ischaemia.

\section{Patients and methods}

Thirty patients were enrolled, but only 23 completed the study: 19 men and four women, aged 40 to 72 (mean 56.2 ) years, who had stable exertional angina of eight to 156 months duration (mean $56 \cdot 2$ ). The other seven patients were excluded at the end of the run in period because of a negative exercise test and were not included in the analysis. All patients had positive exercise tests for myocardial ischaemia ( $\geqslant 1 \mathrm{~mm}$ horizontal or downsloping ST segment depression, measured $60 \mathrm{~ms}$ after the J point) and documented coronary artery disease (five patients with one vessel, 11 patients with two vessel and seven patients with three vessel disease). There had been no change in the frequency, duration, or severity of their angina in the three months before the study. Four patients had suffered a previous $Q$ wave myocardial infarction ( $\geqslant$ six months) before the study. All patients had normal blood pressure, were in sinus rhythm, and had no evidence of heart failure, cardiomyopathy, or valvar heart disease. No patient had evidence of left ventricular hypertrophy or conduction defects on the electrocardiogram, which could have interfered with the interpretation of ST segment changes, and no patient was taking digitalis. 
STUDY PROTOCOL

The protocol was approved by the Hammersmith Hospital research ethics committee and all patients gave fully informed written consent for the study. All patients had already undergone at least two diagnostic treadmill exercise tests. Anti-anginal treatment was discontinued for five days before the study, but patients were free to use sublingual glyceryl trinitrate as required; no exercise test was performed within four hours of the last sublingual treatment with this drug. After a two week single blind run in period with $50 \mathrm{mg}$ of atenolol twice daily, a randomised, double blind, three way cross over study was performed in which $50 \mathrm{mg}$ of atenolol, $20 \mathrm{mg}$ of slow release nifedipine, and their fixed combination were given twice daily for three weeks.

Treadmill exercise testing and 24 hour ambulatory electrocardiographic monitoring were used to detect myocardial ischaemia on day 5 while off treatment and at the end of each treatment period. Patients kept angina diaries throughout the trial period, and were asked to record the number of anginal attacks and their use of glyceryl trinitrate.

\section{EXERCISE TESTING}

Exercise testing was performed with the modified Bruce protocol on a computerised treadmill system (Marquette case 12). Twelve lead electrocardiogram recording and blood pressure measurement (cuff) were taken at rest, at the end of each minute during exercise, at the point of $1 \mathrm{~mm}(0.1 \mathrm{mV}) \mathrm{ST}$ segment depression, at peak exercise, and three and six minutes into the recovery period. Three electrocardiogram leads were monitored continuously before and during exercise and for six minutes into recovery. All tests were performed at the same time of day, four to six hours after the last dose of treatment for each patient. A positive exercise test diagnostic of myocardial ischaemia was defined as horizontal or downsloping ST segment depression $\geqslant 1 \mathrm{~mm}$ measured $60 \mathrm{~ms}$ after the J point with respect to the resting value. The exercise test was stopped in the event of chest pain of moderate severity, ST segment depression $\geqslant 2 \mathrm{~mm}$, or inability of the patient to exercise further.

For all exercise tests, exercise duration (minutes) was measured and heart rate-systolic blood pressure product (beats/min. $\mathrm{mm} \mathrm{Hg}$ ) calculated at $1 \mathrm{~mm} \mathrm{ST} \mathrm{segment} \mathrm{depression} \mathrm{or,}$ when the exercise test was negative at peak exercise, and the value of the rate-pressure product was used as an index of the ischaemic threshold. The reason for stopping the test was noted.

\section{AMBULATORY HOLTER MONITORING}

Ambulatory electrocardiographic recordings were taken from prejelled electrodes, positioned after careful skin preparation to record 2 bipolar leads (modified lead III and an anterior lead), with 2 channel amplitude modulated recorders (Marquette 8500). The anterior lead selected for each patient was that which had no $Q$ wave and had shown maximal
ST segment depression during exercise. All patients were instructed to continue their normal daily activity and to use glyceryl trinitrate only for symptomatic relief. An episode of myocardial ischaemia was defined as horizontal or downsloping ST segment depression or ST segment elevation $\geqslant 1 \mathrm{~mm}(0 \cdot 1 \mathrm{mV})$ at $60 \mathrm{~ms}$ from the $\mathrm{J}$ point, of more than one minute duration and separated from the next episode by at least two minutes of baseline electrocardiogram.

\section{DATA ANALYSIS}

For each exercise test the level of the ST segment, $60 \mathrm{~ms}$ after the J point, was calculated after signal averaging by a computer assisted system in all 12 leads every minute with an accuracy of $0.1 \mathrm{~mm}$. The lead showing the greatest ST segment depression was selected for analysis. The values then measured were: exercise time (minutes) and heart rate-blood pressure product (beats/min. $\mathrm{mm} \mathrm{Hg}$ ) at the start of $1 \mathrm{~mm}$ ST segment depression; if the exercise test was negative, the values were taken at peak exercise. The reason for stopping the test was noted. The exercise tests were analysed by people blinded to the treatment.

The tapes taken during ambulatory electrocardiographic monitoring were analysed (blind as to treatment) with a Marquette Laser Holter System 800 . The total duration of myocardial ischaemia was measured from each 24 hour period.

\section{STATISTICAL ANALYSIS}

The statistical analysis was performed by analysis of variance with pairs compared by $t$ test. A p value $<0.05$ was considered statistically significant. Data are expressed as mean (SEM).

\section{Results}

CONTROLS

The exercise tests in all patients were positive for myocardial ischaemia while they were not taking drugs (control). The exercise test was stopped because of severe chest pain in 14 patients and silent ST segment depression $\geqslant 2 \mathrm{~mm}$ in nine patients. The exercise time and rate-pressure product at $1 \mathrm{~mm} \mathrm{ST}$ segment depression were 7.4 (0.8) $\mathrm{min}$ and 20041 (1046) beats/min. $\mathrm{mm} \mathrm{Hg}$ respectively (fig 1 ). The total ischaemic time during ambulatory monitoring was $69(17 \cdot 1) \mathrm{min}$ (fig 2).

\section{EFFECT OF NIFEDIPINE}

At the end of the period of taking nifedipine, in five patients the exercise test was negative and was stopped due to fatigue. Of 18 patients who had positive test 12 stopped due to chest pain and one due to shortness of breath; the remaining five were stopped due to silent ST segment depression $>2 \mathrm{~mm}$. Compared with the control, the exercise time to $1 \mathrm{~mm} \mathrm{ST}$ segment depression increased from $7 \cdot 4(0.8)$ to $10 \cdot 6(0.7)$ min $(p<0.001)$. This increment was also associated with an increase in the rate-pressure product at $1 \mathrm{~mm} \mathrm{ST} \mathrm{segment} \mathrm{depression} \mathrm{from}$ 
Figure 1 Bar graph of changes in rate-pressure product and exercise time at $1 \mathrm{~mm} S T$ segment depression and number of positive exercise tests for control periods, treatment with atenolol, nifedipine, and the combination of atenolol and nifedipine (mean (SEM)). Atenolo and the combination pressure product and increased exercise time with fewer positive tests compared with nifedipine alone. significantly reduced rate-

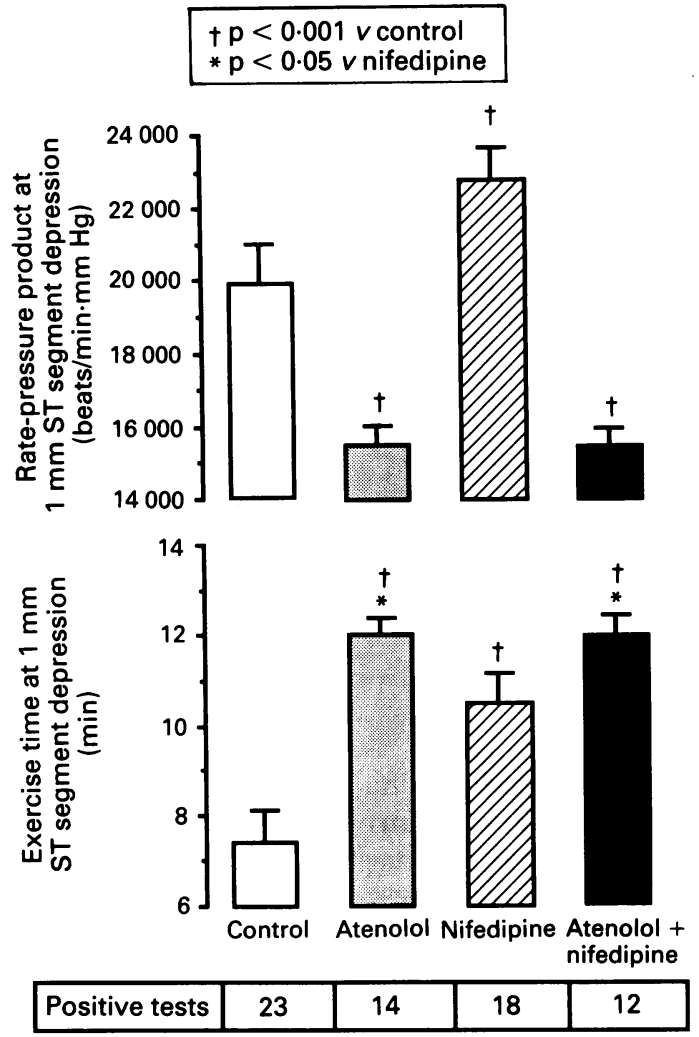

20041 (1046) to 22865 (841) (p < 0.001) (fig 1). The total ischaemic time during ambulatory monitoring was reduced from $69(17 \cdot 1)$ to $37 \cdot 5$ $(9.8) \min (\mathrm{p}<0.01)$ (fig 2 ). The average number of anginal attacks during this period was 5 $(0 \cdot 2)$ and the use of glyceryl trinitrate was 6 (1.9) per week (fig 3).

\section{EFFECT OF ATENOLOL}

At the end of the period of taking atenolol nine patients had a negative exercise test and stopped due to fatigue. Of 14 patients who had positive tests, five were stopped because of chest pain, three due to shortness of breath and the remaining six due to silent $S T$ depression $>2 \mathrm{~mm}$. Atenolol increased exercise time to $1 \mathrm{~mm} \mathrm{ST} \mathrm{segment} \mathrm{depression} \mathrm{from} \mathrm{7.4} \mathrm{(0.8)} \mathrm{to}$ $11.9(0.4) \min (\mathrm{p}<0.001 v$ control; $\mathrm{p}<0.05 v$ nifedipine). This was associated with a reduction in the rate-pressure product at $1 \mathrm{~mm} \mathrm{ST}$ segment depression from 20041 (1046) to 15605 (499) (p < 0.01 v control and nifedipine) (fig 1). The total ischaemic time during ambulatory monitoring was reduced further from $69(17.1)$ to $15.6(5.5) \mathrm{min}$

Figure 2 Bar graph showing mean total duration of ischaemia on ambulatory electrocardiographic monitoring for control periods and during periods and during nifedipine, and combination of atenolol and nifedipine (mean (SEM)). The combination significantly reduced total ischaemic time compared with all other groups.

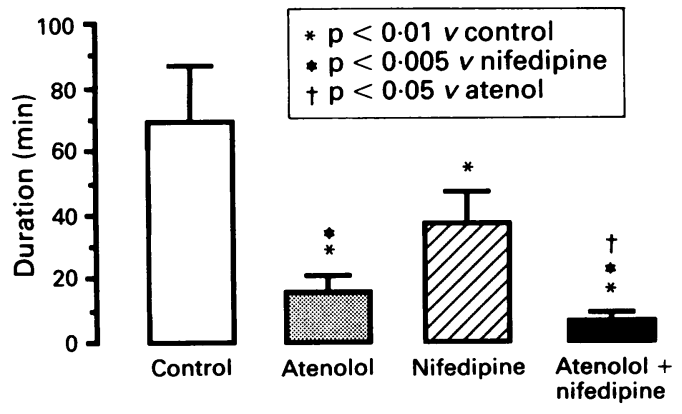

EFFECT OF ATENOLOL, NIFEDIPINE, AND THEIR COMBINATION ON HEART RATE

Compared with the control the resting heart rate was increased by 13 (7) beats/min with nifedipine $(\mathrm{p}<0.001)$ but was reduced by 22 (7) beats/min by atenolol and 19 (7) by the combination $(p<0.001)$. Figure 4 gives the heart rate measured minute by minute during the exercise tests, and shows that nifedipine resulted in a higher heart rate throughout exercise when compared with control, atenolol, and the combination of atenolol and nifedipine. 
Figure 4 Mean heart rate during the exercise test for control periods and during treatment with atenolol, nifedipine, and combination of atenolol and nifedipine. Nifedipine induced a significant reflex increase in resting heart rate compared with the other groups. For each level of exercise, heart rate was significantly lower with atenolol than with nifedipine, and similar to that with atenolol and nifedipine combined.

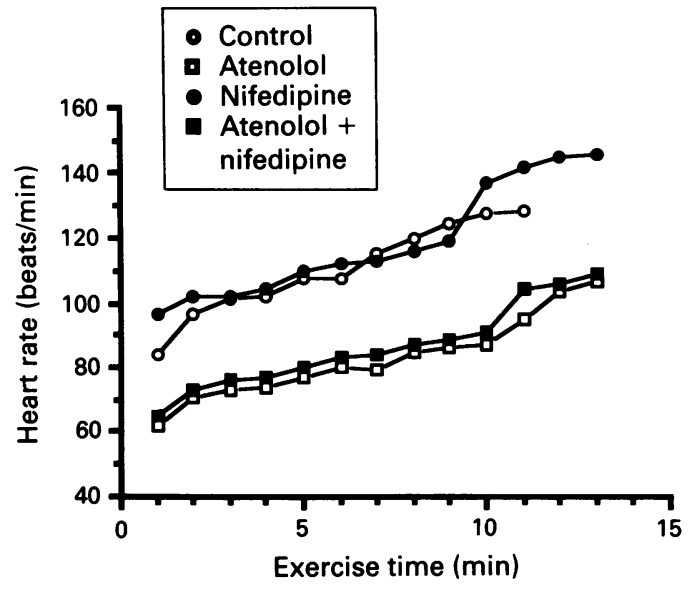

\section{Discussion}

Many of the patients with chronic stable angina often show an unpredictable variability of effort tolerance and even have occasional spontaneous attacks of angina at rest. $^{3}$ In these patients ischaemia can be caused by an increase in myocardial oxygen demand that increases with a rise in the heart rate, contractility, or intraventricular volume and pressure. Alternatively, it can be caused by a reduction in myocardial blood flow due to dynamic constriction at the site of a stenosis of an epicardial coronary artery, ${ }^{4}$ by an increase in coronary arteriolar resistance, ${ }^{5}$ or by an increase in left ventricular wall tension that reduces subendocardial blood flow. ${ }^{6}$ The options for treatment in these patients lie in choosing a drug that acts on all these different factors of myocardial ischaemia. Because one anti-anginal drug usually modifies only a few of these, the addition of a second agent with different pharmacological actions may act in a complementary fashion to control myocardial ischaemia.

This study shows that a fixed combination of atenolol and nifedipine is more effective than either drug alone in reducing ischaemia detected by Holter monitoring during unrestricted daily life. It is also more effective than nifedipine alone in reducing the number of anginal attacks and the use of glyceryl trinitrate. The effects of the combination on exercise induced ischaemia assessed by standardised exercise testing was evident through a significant reduction of the ischaemic threshold compared with control and nifedipine alone, and was similar to that found with atenolol alone. It has also been shown that the combination increases the left ventricular ejection fraction with almost complete abolition of angina pectoris when compared with either drug alone. ${ }^{7}$

In our study nifedipine induced an increase in exercise time to $1 \mathrm{~mm} \mathrm{ST} \mathrm{segment} \mathrm{depres-}$ sion but resulted in a negative exercise test in only five out of the 23 patients. It also had a modest but significant effect on ambulatory ischaemia. It has been shown that nifedipine relieves ischaemia primarily by improving myocardial perfusion by antagonising vasoconstriction at the site of a stentic lesion ${ }^{8}$ or by improving collateral blood flow to ischaemic regions. ${ }^{9}$ The systemic vasodilatory effect of nifedipine also acts to reduce myocardial consumption of oxygen but the peripheral action stimulates reflex action of the sympathetic nervous system. ${ }^{10}$ The resulting increases in heart rate may reduce the antianginal effects of this drug ${ }^{11}$ and account for our finding of only a small beneficial effect.

In our study, atenolol alone significantly increased the exercise time to $1 \mathrm{~mm}$ ST segment depression compared with nifedipine alone and made nine out of 23 exercise tests negative. This was achieved by a reduction of the heart rate at rest and during exercise (patients reached a mean maximum heart rate of only 100 beats/min after exercising for nearly 15 minutes) (fig 4). Atenolol also reduced the total duration of ambulatory ischaemia, further than nifedipine, by $77 \%$ of the control value. It also had a beneficial effect on the number of anginal attacks when compared with nifedipine monotherapy. $\beta$ Blockers are known to relieve ischaemia primarily by decreasing myocardial oxygen demand through inhibition of the rise in heart rate and blood pressure that accompanies submaximal and maximal exercise. ${ }^{12}$ They have been shown in other studies to be effective in reducing ischaemia during ambulatory activities. ${ }^{13}$ The fixed combination of atenolol and nifedipine produced effects during exercise testing similar to those found with atenolol alone, and also produced similar behaviour of the heart rate (fig 4).

In conclusion atenolol and its combination with sustained release nifedipine are equally efficacious in controlling that aspect of stable angina which is due to dynamic exercise alone. This effect is explained by the similar behaviour of heart rate during exercise with both preparations. The additional benefit, however, of the combination on ambulatory ischaemia during unrestricted daily life is consistent with the effect of nifedipine on that component of chronic angina determined by variations in vasomotor tone. The potentially adverse effect of the high resting heart rate induced by nifedipine on the dynamic exercise component of angina is neutralised by its combination with atenolol.

1 Maseri A. The changing face of angina pectoris. Lancet

2 Epstein SE, Talbot TL. Dynamic coronary tone in precipitation, exacerbation and relief of angina pectoris. precipitation, exacerbation and

3 Maseri A. Medical therapy of chronic stable angina pectoris. Circulation 1990;82:2258-62.

4 Gage JE, Hess OM, Murakami T, Ritter M, Grimm J, Krayenbuehli HP. Vasoconstriction of stenotic coronary arteries during dynamic exercise in patients with classic angina pectoris: reversibility by nitroglycerin. Circulation 1986;73:865-75.

5 Cannon RO III, Watson RM, Rosing DR, Epestine SE Angina caused by reduced vasodilator reserve of the small coronary arteries. J Am Coll Cardiol 1983;1:1359-73.

6 Ellis AK, Klocke FJ. Effect of preload of the transmura distribution of perfusion and pressure flow telationship in the canine coronary vascular bed. Circ Res 1980;46:68-77.

7 Findlay IM, MacLeod K, Ford M, Gillen G, Elliott A Dargie HJ. Treatment of angina pectoris with nifedipine and atenolol: efficacy and effect on cardiac function. $B r$ Heart $J$ 1986;55:240-5.

8 Gunther S, Green L, Mulbr JE, Mudge GH Jr, Grossman W. Prevention by nifedipine of abnormal coronary vasoconstriction in patients with coronary artery disease. Circulation 1981;63:849-55. 
9 Rache RJ, Tockman BA. Effect of nitroglycerin and nifedipine on subendocardial perfusion in the presence of a flow-limiting coronary stenosis in the awake dog. Circ Res 1982;50:678-87.

10 Lown B. Introduction. Symposium on nifedipine and calcium flux inhibitors in the treatment of coronary artery
spasm and myocardial ischaemia. Am J Cardiol 1979; 44:780-2.

11 Schanzenbacher P, Liebau G, Deeg P, Kochsiek K. Effect of intravenous and intracoronary nifedipine on coronary blood flow and myocardial oxygen consumption. $\mathrm{Am} \mathrm{J}$ Cardiol 1983;51:712-7.

12 Subramanian VB, Bowles MU, Davies AB, Raftery EB. Calcium channel blockade as primary therapy for stable angina pectoris: a double-blind placebo-controlled comparison of verapamil and propranolol. Am J Cardiol parison of verap

13 Dargie HJ, Lynch PG, Krikler DM, Harris L, Krikler S. Nifedipine and propranolol: a beneficial drug interaction. Am J Cardiol 1981;71:676-82 